\title{
Presentation and Contextualisation in the Online Archive of Internet Art
}

\author{
Lozana Rossenova \\ London South Bank University \\ 103 Borough Road \\ London SE1 OAA, UK \\ mehandzl@Isbu.ac.uk
}

\begin{abstract}
This is a presentation of the initial findings of a collaborative practice-based $\mathrm{PhD}$ research project at London South Bank University (London, UK) and Rhizome (New York, US). The project analyses challenges associated with presenting and contextualising internet art in an online archive through the lens of user interaction design. The term internet art is contentious. It is used here within the definitions given by Stallabrass (2003) and Greene (2004). This is to distinguish it from the earlier narrower definition of the term net.art or the later expanded term internet-based art.
\end{abstract}

\section{INTRODUCTION}

Changes in web standards and developments in new patterns of interaction between users and computers create multiple challenges for digital archives and long-term provision of access to digital artefacts of cultural value (Wray et al. 2013). Curation and presentation in archives of internet art, in particular, pose multiple sets of problems closely associated with the question of what constitutes the art object. Internet art works are rarely single digital objects, but rather assemblages, dependent on specific software/hardware environments. They oftentimes change over time and require specific user input to be performed.

This practice-based research project seeks to better understand problems associated with the archiving of internet art and to address the question of how artworks can be made accessible to the public in their native environment - online - while enabling meaningful interactions between users and the archive. The primary case study of this research is a redesign of the user interaction framework of the ArtBase, the online archive maintained by Rhizome, a leading international arts organisation dedicated to curating and archiving internet art.

\section{CASE STUDY - THE ARTBASE}

In the fields of user experience design and human computer interaction $(\mathrm{HCl})$, there has been substantial research carried out around issues of discoverability, accessibility and usability in digital archives, but the studies have focused primarily on archives with text- or image-based documents (Wray et al. 2013, Johnson 2008). Presentation and contextualization, as well as models for user interaction in archives of more complex digital artefacts, however, have been discussed much less, particularly from the point of view of the user's experience.

Like complex computer games, time-based media and performance art, internet art spans beyond the boundaries of a single object and can become 'diffuse' (Fino-Radin 2011). Artworks can reference external, dynamic and real-time data sources, or exist across multiple locations and platforms. Capturing the context of multiple layers of dependencies on software and hardware components, as well as various user interactions, poses challenges to existing digital presentation frameworks. Documenting in metadata and/or indicating through interface design elements a variety of issues specific to internet art archives, including unsupported browser plug-ins, changes in web languages or syntax, interaction models which develop over time and/or over various platforms, among others, remain yet to be fully resolved.

Rhizome has recognised the inherent vulnerability of internet art since its inception as an organisation and community-building platform in 1996. The ArtBase was established in 1999 as an online space to showcase and archive internet art, as well as to build a community and a discourse around the works (Fino-Radin 2011). Initial strategies towards presentation of artworks in the ArtBase followed concurrent developments in the fields of interaction design and digital preservation, but the growing number and variety of artworks in the archive presented multiple challenges. The current 
archival framework is no longer able to provide adequate access to many the historic artworks, and increasingly the focus of preservation efforts at Rhizome has been on building tools to support the presentation of complex artworks with multiple dependencies.

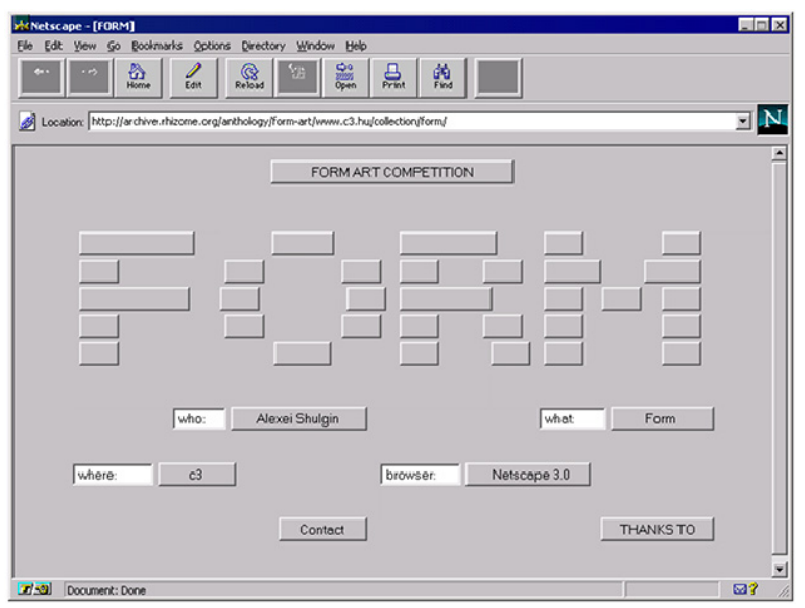

Figure 1: An emulated presentation of Alexei Shulgin's Form Art in Rhizome's Net Art Anthology.

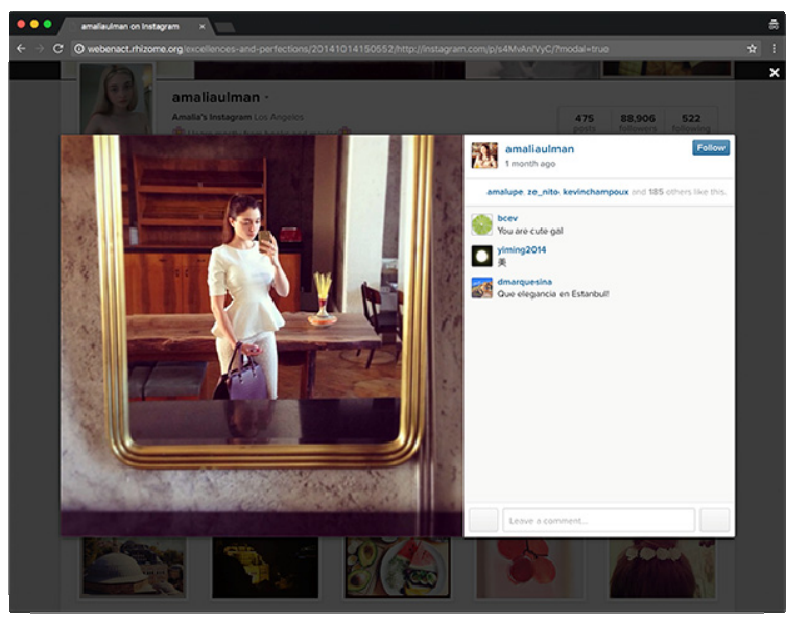

Figure 2: An archived version of Amalia UIman's Instagram performance, Excellences \& Perfections.

Some of the earlier limitations in preservation strategies such as migration and emulation with regards to the presentation of internet art online, have been addressed to some degree by recent developments in browser-based emulation and web archiving tools. The remote browsing technology, first introduced in Rhizome's Oldweb.today project to emulate old browser environments, has facilitated the online restoration and reperformance of historic internet art works in contemporaneous environments (Figure 1). Furthermore, the capacity to create high-fidelity archives of the dynamic web with Rhizome's browser-based archiving tool, Webrecorder, has enabled the preservation of artworks utilising thirdparty web services (Figure 2). These more recent preservation efforts, however, have not yet been integrated with the current ArtBase data structure.

Considering how recent developments in preservation strategies can be better integrated in the ArtBase archival system and the interface design framework requires further user research and design exploration.

\section{METHODOLOGY}

This project is multidisciplinary, combining qualitative research methods from the fields of the digital humanities, information sciences and $\mathrm{HCl}$. Following common $\mathrm{HCl}$ research approaches, the research work involves ethnographic observation and contextual inquiry. Additionally, this research project follows the concept of the design artefact as knowledge carrier (Stolterman \& Wiberg 2010) and the paradigm of critical design practice (Gaver 2012), wherein implicit design theory should be allowed to 'emerge' through situated design practice, instead of constructing design artefacts to explicitly test or produce theory.

\section{CONTRIBUTION TO THE FIELD}

Existing research in the fields of digital preservation and user interaction design have not yet addressed many of the specific challenges associated with presenting and contextualising internet art works in an online archive. This project applies methods from $\mathrm{HCl}$ research to study user behaviours and carry out concept-driven design exploration to develop a design framework supporting increasingly complex and 'diffuse' artworks and their related user interaction models.

\section{REFERENCES}

Fino-Radin, B. (2011) Digital Preservation Practices and the Rhizome ArtBase. Rhizome. http://media.rhizome.org/artbase/documents/Digital -Preservation-Practices-and-the-RhizomeArtBase.pdf (accessed 24 February 2017).

Gaver, W. (2012) What should we expect from research through design? In Proceedings of the SIGCHI Conference on Human Factors in Computing Systems (CHI '12), Austin, Texas, 5-10 May, pp. 937-946.

Greene, R. (2004) Internet Art. London: Thames \& Hudson.

Johnson, A. (2008) Users, use and context: supporting interaction between users and digital archives. In Craven, L. (ed.) What are Archives? Cultural and Theoretical Perspectives: A Reader. 
Aldershot, pp.145-166. Hampshire, England; Burlington, Vermont: Ashgate.

Stallabrass, J. (2003) Internet Art: The Online Clash of Culture and Commerce. London: Tate Publishing.
Stolterman, E. and Wiberg, M. (2010) Conceptdriven interaction design research. HumanComputer Interaction, 25(2) ), pp.95-118, June.

Wray, T., Eklund, P., and Kautz, K. (2013) Pathways through information landscapes: Alternative design criteria for digital art collections. In Proceedings of the International Conference on Information Systems (ICIS 2013). pp. 2894-2914. 